\title{
THE 'DETWISTED’ PROMINENCE OF SEPTEMBER 12, 1966
}

\author{
BORIS VALNíČEK \\ (Astronomical Institute, Czechoslovak Academy of Sciences, Ondřejov, C.S.S.R.)
}

\begin{abstract}
The eruptive prominence of September 12, 1966, is described. Detwisting of two filaments is demonstrated and compared with similar phenomena and with tornado prominences. A short discussion of mechanisms of the origin and subsequent development of the phenomenon is given.
\end{abstract}

\section{Description of the Phenomenon}

On September 12, 1966, 9.25 UT we saw a bright prominence on the East limb. During the next 2-3 min this prominence weakened, but in the lower part near the limb we observed a brightening which started to grow. After $9 \cdot 30$ UT it was clear that an interesting process had started. The outer part of the prominence was still growing, and at 9.45 a complicated inner structure began to develop.

After 9.50 UT we saw a very bright phenomenon on the coronagraph observations made in the $\mathrm{H} \alpha$ line with an $8 \AA$ passband filter. It was composed of two interwoven spirals which straightened (detwisted) during the next $15 \mathrm{~min}$. At 10.24 UT we found only one prominence filament remaining close to the limb. This filament did not undergo important and fast changes. The visibility of this prominence deteriorated during the day, but it remained visible.

The following day, September 13, we observed on the East limb a large spot group, with a filament crossing the limb. After this the group with the filament could be followed throughout the passage across the disk.

\section{Analysis of the Observations}

At first we studied motion effects in the prominence. Linear displacements of the mass are presented in the velocity curve, which shows three important phases:

(a) The sharp velocity maximum at $\mathbf{9 \cdot 4 0}$ UT was in coincidence with strong radio emission at the wavelengths $3 \cdot 2,37,56$, and $115 \mathrm{~cm}$.

(b) At 9.50 UT after a sudden decrease of the velocity there followed a new rise with maximum value, coinciding also with a radio-noise maximum at a wavelength of $115 \mathrm{~cm}$.

(c) Before 10.00 UT a small decrease can be found followed by a fast rise to the maximum value. 

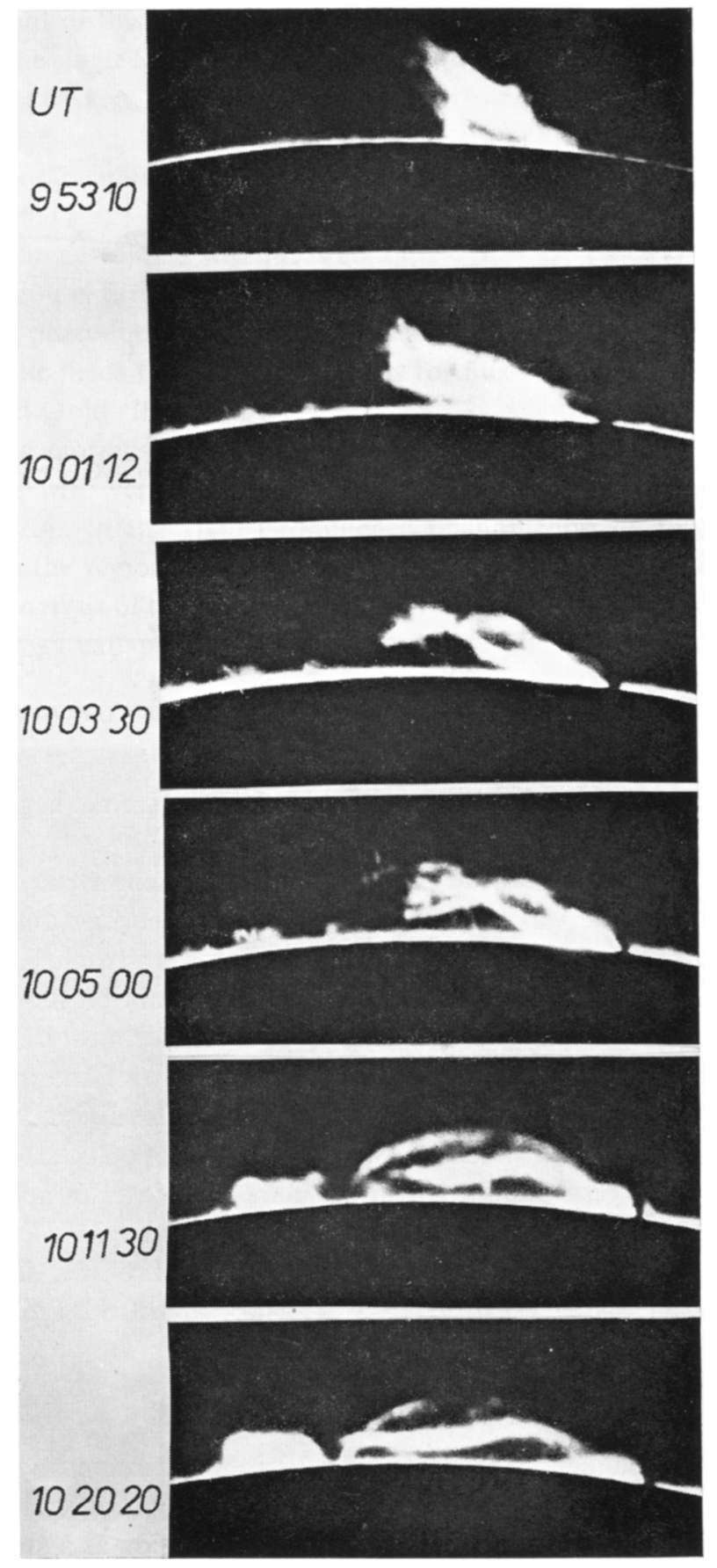

FIG. 1. Coronagraph observations of the detwisting phase of September 12, 1966. Ondrejov Observatory photograph. 


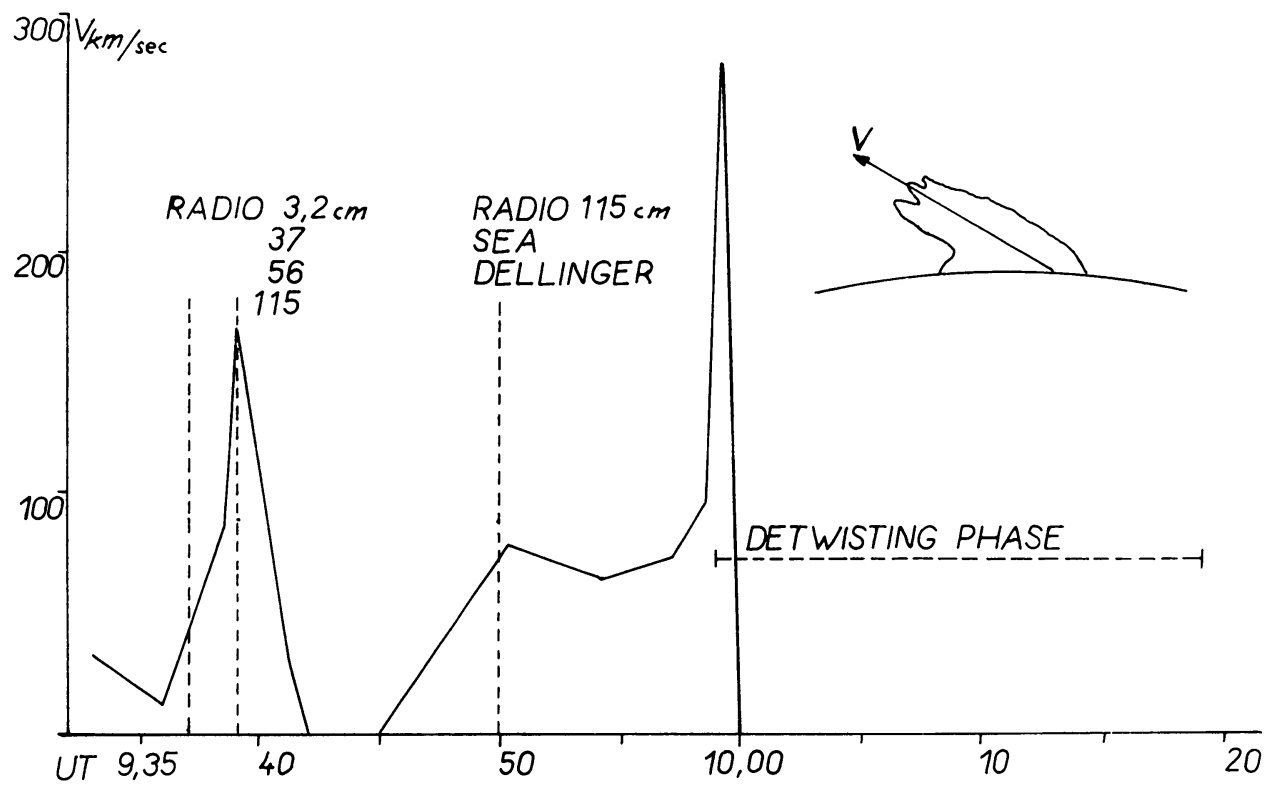

FIG. 2. Graphic representation of the velocity changes (full curve).

After this last phase the detwisting of the interwoven spirals began. This finished at $10 \cdot 20 \mathrm{UT}$.

It seems that the most energetic phase of the phenomenon was at 9.35-9.37 UT, when the radio emission was very important at all frequencies and a strong X-ray burst can be surmised. At this time the phenomenon was situated in deeper levels of the corona and in the chromosphere, as can be seen clearly on the photographs.

It is interesting that the second phase at $9.50 \mathrm{UT}$ was the most important from the geophysical point of view. It was the time of maximum of the greatest effects of motion and very rapid brightening of the prominence. The division into two twisted filaments also started at this time.

For this period we also obtained with our flare-spectrograph a series of spectrograms clearly demonstrating for two times - 9.53 UT and 9.59 UT - Doppler shifts of both senses. This could be confirmation of the existence of a detwisting motion of both filaments. Doppler velocities measured here are of the order $200 \mathrm{~km} / \mathrm{sec}$.

\section{Conclusions}

The observed phenomenon of September 12 is most interesting because of the detwisting process of two spiral-form filaments. Similarly of interest is the fact that one of the detwisted spirals remained above the active region as a stationary filament during the entire passage of the region across the disk. 
The development of this phenomenon resembles the mechanism tested by Bostick (1958) by firing one single 'plasmoid' across a magnetic-field. The result is of the same shape as the second stage of the prominence which was observed. From this point of view we can suppose that a very energetic process in the low chromosphere at the moment of maximum radio emission, $9.40 \mathrm{UT}$, caused the emission of matter which provoked the second stage - the double-spiral system of the prominence after $9 \cdot 50 \mathrm{UT}$.

This thought is in agreement with ideas contained in the work of Carmichael (1963) and our observation is further evidence for this.

The detwisting phenomenon can be explained as the product of an unstable state in twisted magnetic fields following conditions for flux-tube instability, described by Alfvén (1955) and Gold (1963).

Such detwisting motions seem to be very general phenomena, especially in cases closely connected with very energetic eruptive processes. We have observed a very similar process, e.g., in the great prominence phenomenon of July 11, 1966. This effect occurred in the region of the proton-flare, included in the PFP, and we have given a detailed analysis of this phenomenon in the PFP-papers (Valníček et al., 1968).

A certain analogy can probably be found in the phenomenon of Petit's (1950)

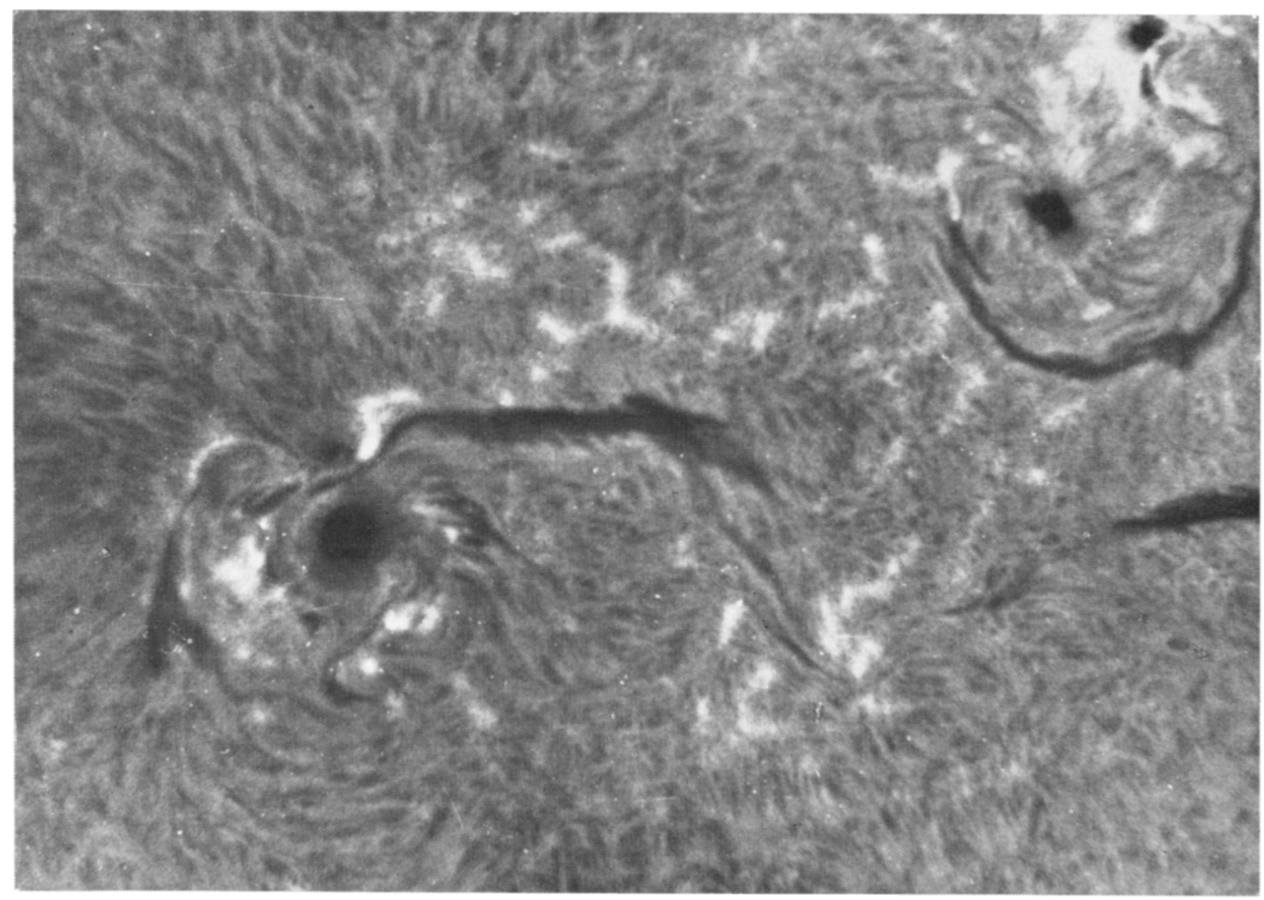

FIG. 3. Detail of the chromospheric structure on September 19, 7.50 UT. The orientation is the same as in Figure 1. Note the whirling structure of the filament. Ondřejov Observatory photograph. 
tornado prominences. Only the duration of both effects seems to be important in judging the difference between them: the lifetime of tornado prominences is of the order of days or weeks, while for the eruptive type described here it is of the order of 1 hour. But it seems that the filament, observed during its passage on the disk after September 12, resembles very closely the one observed by Richardson and Hickox and described in Petit's paper.

So it can be concluded that the tornado prominence is the later stage of a detwisted flare prominence of the type observed by us on September 12, 1966. This prominence - in its filament form - is also studied in the present volume by Sara Smith and by Rayrole (see pp. 267 and 134 respectively).

The future study of this active center could be very interesting in connection with other materials. The magnetic-field chart, presented by Rayrole, gives only the center of the active region, the position of the Northern part of the filament which could be seen on September 19. Nothing is known about the flare stage of the active region. But because the spots have not been observed here, the probability of obtaining a detailed magnetic-field chart is very small. Only the chaotic chromosphere structure indicates the present turbulent motions and the complicated magnetic structure.

\section{References}

Alfvén, H. (1955) in Cosmical Electrodynamics, p. 117.

Bostick, W.H. (1958) Rev. mod. Phys., 30, 1090.

Carmichael, H. (1963) AAS-NASA Symposium on the Physics of Solar Flares, p. 451.

Gold, T. (1963) AAS-NASA Symposium on the Physics of Solar Flares, p. 389.

Petit, E. (1950) Publ. astr. Soc. Pacific, 62, 144.

Valnícek, B., Godoli, G., Mazzucconi, F. (1968) IQSY-Annals, PFP-Project. 